\title{
A New Variable Exponential Power Reaching Law of Complementary Terminal Sliding Mode Control
}

\author{
Feng Xu $\mathbb{D}^{1},{ }^{1}$ Na An, ${ }^{2}$ Jianlin Mao $\mathbb{D}^{1},{ }^{1}$ and Shubo Yang ${ }^{1}$ \\ ${ }^{1}$ School of Information Engineering and Automation, Kunming University of Science and Technology, Kunming 650500, China \\ ${ }^{2}$ Kunming Precision Machinery Research Institute, Kunming 650032, China
}

Correspondence should be addressed to Jianlin Mao; 1318524654@qq.com

Received 20 August 2020; Revised 7 September 2020; Accepted 21 September 2020; Published 14 October 2020

Academic Editor: Shubo Wang

Copyright $\odot 2020$ Feng Xu et al. This is an open access article distributed under the Creative Commons Attribution License, which permits unrestricted use, distribution, and reproduction in any medium, provided the original work is properly cited.

In this article, a new nonlinear algorithm based on the sliding mode control is developed for the ball and plate control system to improve dynamic response and steady-state tracking accuracy of the control system. First, a new sliding mode reaching law is proposed, variable exponential power reaching law (VEPRL), which is expressed in two different forms including a nonlinear combination function term and a variable exponential power term, so that it can be adjusted adaptively according to the state of the system by the variable exponential power reaching term during the reaching process. The computation results show that it can not only effectively weaken the chattering phenomenon but also increase the rate of the system state reaching to the sliding mode surface. Moreover, it has the characteristic of global finite-time convergence. Besides, a complementary terminal sliding mode control (CTSMC) method is designed by combining the integral terminal sliding surface with the complementary sliding surface to improve the convergence rate. Based on the proposed VEPRL and CTSMC, a new sliding mode control method for the ball and plate system is presented. Finally, simulation results show the superiority and effectiveness of the proposed control method.

\section{Introduction}

Sliding mode control is essentially a nonlinear control method with control discontinuity. Due to its superior robustness, low model dependence, and flexible parameters, it has been widely used in many control fields [1-5]. However, in the actual engineering application process, the chattering problem may cause high-frequency oscillation of the system and cause instability. How to eliminate the chattering problem and improve the convergence rate is still a hot topic in the sliding mode control community. In this line, many research works have been carried out, such as boundary layer method [6], intelligent control method [7-10], high-order sliding mode control method [11], complementary sliding mode control method [12], repetitive learning control method [13], and reaching law method [14]. The approaching law method can improve the dynamic convergence quality of the approaching process of the system and effectively solve the chattering problem. Therefore, since Gao [15] proposed the exponential reaching law, power reaching law, and constant reaching law, many researchers have also carried out research to study the reaching law and have obtained many novel and improved reaching laws [16-20]. In [16], Yu et al. proposed a fast power reaching law, which shortens the reaching time by linearly combining the exponential reaching law and the single power reaching law. In [17], a novel exponential reaching law including the exponential term function of the sliding mode variable is proposed. The exponential term can smoothly adapt to the change of the sliding mode variable and can effectively reduce the chattering phenomenon. By increasing the order of reaching law, in [18], Mei et al. proposed a double-power sliding mode reaching law, which was applied to the robot tracking problem. Although this idea can increase the reaching speed, it did not give a qualitative analysis of the reaching law. In the study of Zhang et al. [19], a multipower sliding mode reaching law was proposed on the basis of double-power sliding mode reaching law with qualitative analysis, which can improve the system dynamic response speed and effectively suppress 
the chattering phenomenon. In [20], a new discrete reaching law sliding mode control method was proposed for an uncertain discrete-time system, and the quasi-sliding-mode domain was improved by redefining the change rate as the second-order difference of system uncertainties and adopting the continuous-approximate function. Su et al. [21] proposed a complementary sliding mode control (CSMC), which effectively weakened the chattering phenomenon, reduced system tracking errors, and improved the robustness of the system. In [22], a control scheme combining the complementary sliding mode control and Elman neural network, and developed a control of the permanent magnet linear servo motor, has good dynamic response and steadystate control accuracy.

To address the approaching rate of the system tracking error and weaken the chattering problems, a new sliding mode control method is proposed for the ball and plate system in this paper. The main contributions of the paper are summarized as follows. (1) A new variable exponential power reaching law is proposed, which can adaptively adjust the exponential parameter at different stages of the control system response so that the approaching process of the system is accelerated. We prove that it can converge to the equilibrium point in a finite time. (2) Combined with the complementary terminal sliding mode control, a complementary terminal sliding mode control method is proposed, with which the controlled system can have a better dynamic response and control accuracy. (3) Based on the above two methods, we present a new controller for the ball and plate system, which shows good control performance.

\section{Design of Variable Exponential Power Reaching Law}

The variable exponential power reaching law is designed as

$$
\dot{s}=-k_{1} \mathrm{fal}(s, \alpha, \delta)-k_{2}|s|^{\beta} s,
$$

with

$$
\begin{gathered}
\beta=\operatorname{asgn}(|s|-1), \\
\operatorname{sgn}(x)= \begin{cases}1, & x>0, \\
-1, & x \leq 0,\end{cases} \\
\operatorname{fal}(s, \alpha, \delta)= \begin{cases}|s|^{\alpha} \operatorname{sign}(s), \quad|s|>\delta, \\
\frac{s}{\delta^{1-\alpha}}, & |s| \leq \delta,\end{cases}
\end{gathered}
$$

where $k_{1}>0, k_{2}>0, \alpha=1.5, a=0.5$, and $\delta=1$. By analyzing the reaching law stated in equation (1), the reaching law with a variable exponential power term has a strong adaptive ability. fal $(s, \alpha, \delta)$ is a nonlinear combination piecewise function. By designing appropriate parameters and according to the change of sliding mode variable $s$, equation (1) is equivalent to the following expression:

$$
\begin{cases}\dot{s}=-k_{1}|s|^{\alpha} \operatorname{sign}(s)-k_{2}|s|^{\beta} s, & |s|>1, \\ \dot{s}=-k_{1} \frac{s}{\delta^{1-a}}-k_{2}|s|^{\beta} s, & |s| \leq 1 .\end{cases}
$$

When $|s|>1$, all terms play the role; thus, reaching law (1) has a higher reaching speed than the double-power reaching law. When $|s| \leq 1$, the reaching law becomes a kind of fast power reaching law to ensure that the system state reaches the sliding mode surface in finite time. When $s \longrightarrow 0^{+}$or $s \longrightarrow 0^{-}$, there is $\dot{s} \longrightarrow 0$, chatter hardly occurs. Therefore, whether the system is far away from or near the sliding surface, the reaching law can not only reduce the chattering but also have a fast convergence and make the system state from any position to reach the sliding mode surface in finite time.

\section{Characteristic Analysis}

3.1. Convergence Time Analysis. The existence condition of sliding mode is the premise of the sliding mode control. The motion of the system must tend to switch at the surface $s=0$, i.e., the existence and accessibility condition must be satisfied, $s \dot{s}<0$ [23].

Theorem 1. For the system described in equation (1), the accessibility condition is satisfied.

Proof. A Lyapunov function is considered:

$$
V=\frac{1}{2} s^{2} .
$$

Combining with (1), the time derivative of $V$ is given by

$$
\begin{aligned}
& \dot{V}=s \dot{s}=-k_{1} s f a l(s, \alpha, \delta)-k_{2}|s|^{\beta} s^{2}, \\
& \dot{V}=s \dot{s}= \begin{cases}-k_{1}|s|^{\alpha+1} \operatorname{sign}(s)-k_{2}|s|^{\beta} s^{2}, & |s|>1, \\
-k_{1} \frac{s^{2}}{\delta^{1-a}}-k_{2}|s|^{\beta} s^{2}, & |s| \leq 1,\end{cases}
\end{aligned}
$$

where $k_{1}>0, k_{2}>0$, and then $\dot{V}<0$, and the accessibility condition is satisfied. The system state $s$ can reach the equilibrium point $s=0$.

Theorem 2. For reaching law (1), the system states $s$ and $\dot{s}$ can converge to the equilibrium zero in a finite time, that is, $s=\dot{s}=0$ after a finite time.

Proof. Suppose the initial condition $s(0)=s_{0}>1$, and the converge process of the system is divided into two stages, namely:

(1) The first stage: $s(0)=s_{0} \longrightarrow s\left(t_{1}\right)=1$, in this case, $\alpha=1+\beta=1.5$, and the reaching law can be expressed as 


$$
\dot{s}=-\left(k_{1}+k_{2}\right) s^{\alpha}
$$

By integrating the two sides of equation (6), we have

$$
\int_{0}^{t_{1}} \mathrm{~d} t=\int_{s_{0}}^{1}-\frac{1}{\left(k_{1}+k_{2}\right) s^{\alpha}} \mathrm{d} s
$$

Therefore, the time $t_{1}$ used for $s=s_{0} \longrightarrow s=1$ can be obtained as

$$
t_{1}=\frac{1-s_{0}^{1-\alpha}}{\left(k_{1}+k_{2}\right)(\alpha-1)} \text {. }
$$

(2) The second stage: $s=1 \longrightarrow s\left(t_{2}\right)=0$, in this case, fal $(s, \alpha, \delta)=s, 1-\beta=0.5$, and the reaching law can be expressed as

$$
\begin{aligned}
\dot{s} & =-k_{1} s-k_{2} s^{1-\beta}, \\
\dot{s}+k_{1} s+k_{2} s^{1-\beta} & =0 .
\end{aligned}
$$

By solving differential equation (10), the time $t_{2}$ used for $s=1 \longrightarrow s=0$ can be obtained as

$$
t_{2}=\frac{1}{\beta k_{1}} \ln \left(\frac{k_{1}+k_{2}}{k_{1}}\right) \text {. }
$$

In summary, combining equations (8) and (11), the total time for the system to move from any initial state $s=s_{0}$ to the sliding surface $s=0$ is $T$, which can be calculated by

$$
T=t_{1}+t_{2}=\frac{1-s_{0}^{1-\alpha}}{\left(k_{1}+k_{2}\right)(\alpha-1)}+\frac{1}{\beta k_{1}} \ln \left(\frac{k_{1}+k_{2}}{k_{1}}\right) .
$$

When the initial state of the system is $s(0)=s_{0}<0$, the proof is the same as the above one; that is, the system can reach equilibrium state from any initial position in finite time. Furthermore, according to equation (1), when $s=0$, it can be got that $\dot{s}=0$.

3.2. Analysis of Steady-State Error Bound. When there is an uncertain bounded disturbance in the system, the state variables of the system in equation (1) could converge to a neighborhood of the equilibrium point in a finite time; that is, the system has a ultimate convergence bound.

For this purpose, we consider the following uncertain nonlinear system:

$$
\dot{s}=-k_{1} \mathrm{fal}(s, \alpha, \delta)-k_{2}|s|^{\beta} s+d .
$$

Theorem 3. For system (13), assuming that the uncertain disturbance $d$ is bounded, that is, $|d| \leq D, D>0$, then the system can converge to a small set around zero in finite time, and there is a steady-state error bound $|s|=\left(D / k_{2}\right)^{(1 / 1-\beta)}$.
Proof. Set a Lyapunov function as

$$
V=\frac{1}{2} s^{2}
$$

Then, we have

$$
\begin{aligned}
\dot{V} & =s \dot{s} \\
& =s\left(-k_{1} \mathrm{fal}(s, \alpha, \delta)-k_{2}|s|^{\beta} s+d\right) \\
& \leq-k_{1} s \mathrm{fal}(s, \alpha, \delta)-k_{2}|s|^{\beta} s^{2}+|s| D .
\end{aligned}
$$

When $|s|>1$, we know

$$
\dot{V}=s \dot{s} \leq-\left(k_{1}+k_{2}\right)|s|^{\alpha+1}+|s| D .
$$

Therefore, in equation (16), when $D \leq\left(k_{1}+k_{2}\right)|s|^{\alpha}$, the sliding mode reaching law in this paper can ensure that the state of the system is convergent in the stage of reaching motion.

When $|s| \leq 1$, it follows

$$
\begin{aligned}
\dot{V} & =s \dot{s} \leq-k_{1} s^{2}-k_{2}|s|^{2-\beta}+|s| D, \\
\dot{V}+k_{1} s^{2} & =\dot{V}+2 k_{1} V \leq-k_{2}|s|^{2-\beta}+|s| D .
\end{aligned}
$$

Therefore, when $|s| \geq\left(D / k_{2}\right)^{(1 / 1-\beta)}$, then $\dot{V}+2 k_{1} V \leq 0$. According to reference [25], the system converges in finite time, and the region $|s| \geq\left(D / k_{2}\right)^{(1 / 1-\beta)}$ is a finite-time convergence region, i.e., there exists a steady-state error bound of $|s| \geq\left(D / k_{2}\right)^{(1 / 1-\beta)}$.

3.3. Comparative Analysis of Convergence Performance. In this section, several reaching laws are employed for convergence analysis and compared with the proposed variable exponential power reaching law (VEPRL).

Consider the following system:

$$
\dot{s}=u+d(t)
$$

where $u$ is the control input, $d(t)$ is the disturbance, and $s=c e+\dot{e}$ is the traditional linear sliding surface.

In Figure 1, PRL is the power reaching law [15], ERL denotes the novel exponential reaching law [16], and DPRL indicates the double-power combination function reaching law [24].The parameters of VEPRL are set as $k_{1}=40, k_{2}=60, \alpha=1.5, \delta=1$, and $a=0.5$; the form of PRL is $\dot{s}=-k|s|^{\alpha} \operatorname{sign}(s)$, and the parameters are given as $k=60$ and $\alpha=0.5$; the form of DPRL is $\dot{s}=-k_{1}$ $\mathrm{fal}(s, \alpha, \delta)-k_{2}|s|^{\beta} \operatorname{sign}(s)$, and the parameters are given as $k_{1}=40, k_{2}=60, \alpha=1.5, \beta=0.5$, and $\delta=1$; the form of ERL is $\dot{s}=-\left(k /\left(\Delta+(1-\Delta) e^{-\mu|s|^{w}}\right)\right) \operatorname{sign}(s)$, and the parameters are given as $k=40, \Delta=0.4, \mu=10$, and $w=3$; when system (18) has no disturbance, that is, $d(t)=0$, all the initial values are set as $s_{0}=50$.

Due to the different structures of the reaching laws, to a certain extent, similar parameter settings show the convergence performance of sliding mode variable $s$. When it is far away from the sliding mode surface, the reaching law is a combination of two power reaching laws, which accelerates the approach speed. When nearing the sliding surface, the 


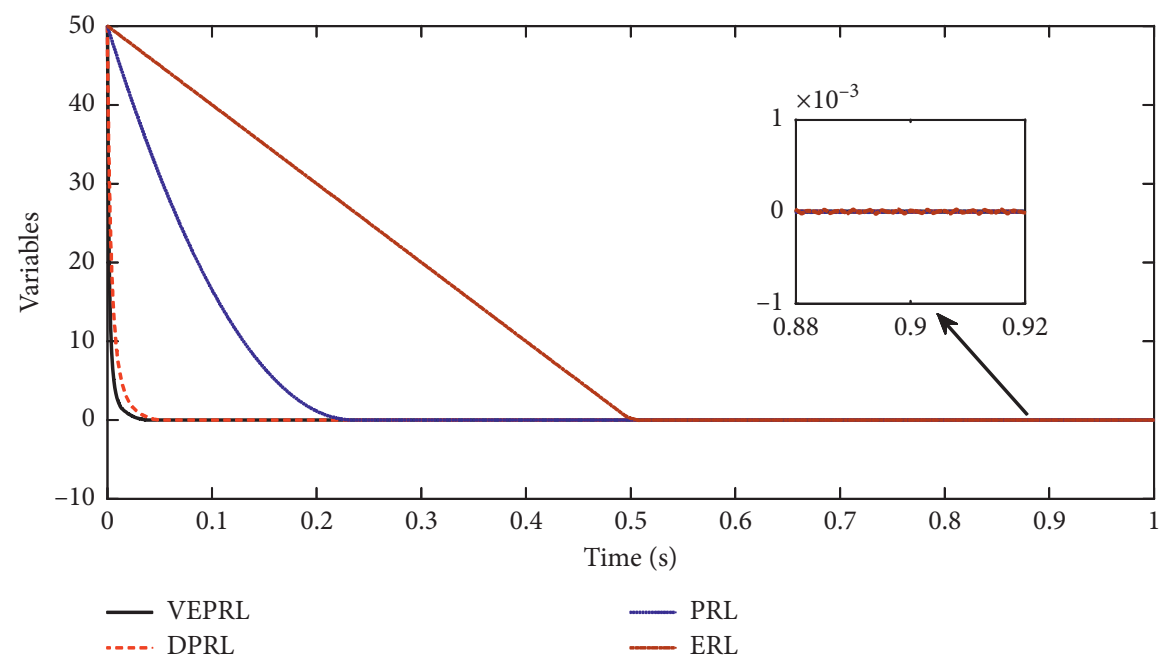

Figure 1: The convergence of different reaching laws.

reaching law is composed of exponential reaching law and power reaching law to ensure that the reaching law can reach the sliding surface in a finite time. By the nonlinear combination piecewise function and the exponential power term to change adaptively at different stages, the reaching law can reach the sliding surface faster. The convergence performance verifies the effectiveness of reaching law (1). From Figure 1, it can be seen that the variable exponential power law has faster convergence characteristics and can reach the sliding surface faster. Similarly, without the interference, the convergence characteristics and performance of other reaching laws can also meet certain requirements.

\section{A Simulation Example}

4.1. Ball and Plate System Mathematical Model. The ball and plate system is a typical control object in control theory research which can test effectiveness of various control algorithms. The ball and plate system is a underactuated, strong coupling, and multivariable system, it extracts the ball position from camera or touch screen and then gives feedback to the controller, and the controller controls the rotation angle of the motor to adjust the tilt angle of the plate, thus realized trajectory tracking control. This paper

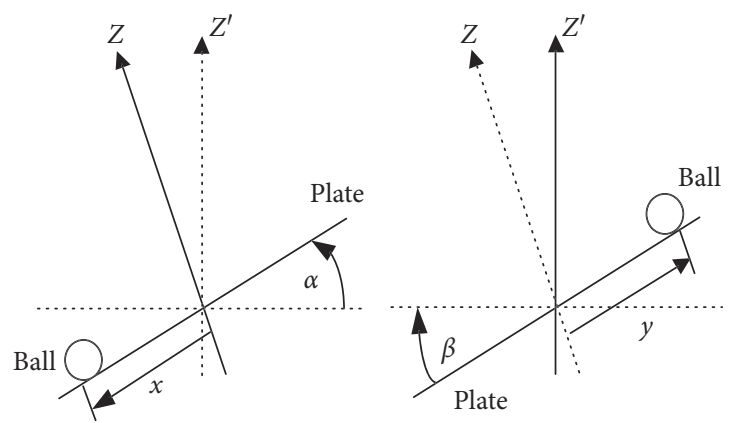

FIgURE 2: Coordinate system of the ball and plate system.

takes the ball and plate system as the control object to verify the proposed sliding mode control method.

Assuming that all frictional forces are ignored, the ball is always in contact with the plate; the area and rotation angle of the plate are unlimited, and the ball does not slide on the plate; the plate is symmetrical about the support point in the $X$ and $Y$ axis directions, and the ball revolves around its balance axis.

From Figure 2, according to the Lagrange equation, the state space equation of the ball and plate system is shown in the following equation:

$$
\left\{\begin{array}{l}
\left(m+\frac{I_{b}}{r_{b}^{2}}\right) \ddot{x}-m\left(x \dot{\alpha}^{2}+y \dot{\alpha} \dot{\beta}\right)+m g \sin \alpha=0, \\
\left(m+\frac{I_{b}}{r_{b}^{2}}\right) \ddot{y}-m\left(y \dot{\beta}^{2}+x \dot{\alpha} \dot{\beta}\right)+m g \sin \beta=0, \\
\left(I_{b}+I_{p}+m x^{2}\right) \ddot{\alpha}+m(x y \ddot{\beta}+\dot{x} y \dot{\beta}+x \dot{y} \dot{\beta})+2 m x \dot{x} \dot{\alpha}+m g x \cos \alpha=\tau_{x}, \\
\left(I_{b}+I_{p}+m y^{2}\right) \ddot{\beta}+m(x y \ddot{\alpha}+\dot{x} y \dot{\alpha}+x \dot{y} \dot{\alpha})+2 m x \dot{x} \dot{\beta}+m g y \cos \beta=\tau_{y},
\end{array}\right.
$$


where $m$ and $r_{b}$ are the mass and radius of the ball, $g$ is the acceleration of gravity, $I_{b}$ and $I_{p}$ are the moment of inertia of the ball and disc, $\tau_{x}$ and $\tau_{y}$ are the torque of the disc in $X$ and $Y$ directions, $\alpha$ and $\beta$ are the inclination angles of the disc in $X$ and $Y$ directions, and $x$ and $y$ are the coordinates of the ball in $X$ and $Y$ directions.

In this paper, according to the assumption, we will linearize the ball and plate system model (20). The tilt angles $\alpha$ and $\beta$ of the disc are selected as the input values $u_{x}$ and $u_{y}$ of the ball and plate system. Take the position and speed of the ball in the $X$ and $Y$ axis directions as the state variables of the system, and let $x_{1}=x, x_{2}=\dot{x}, x_{3}=y$, and $x_{4}=\dot{y}$; the state space expression of the ball and plate system is

$$
\begin{aligned}
{\left[\begin{array}{c}
\dot{x}_{1} \\
\dot{x}_{2} \\
\dot{x}_{3} \\
\dot{x}_{4}
\end{array}\right] } & =\left[\begin{array}{c}
x_{2} \\
0 \\
x_{4} \\
0
\end{array}\right]+\left[\begin{array}{cc}
0 & 0 \\
-\lambda g & 0 \\
0 & -\lambda g \\
0 & 0
\end{array}\right]\left[\begin{array}{l}
u_{x} \\
u_{y}
\end{array}\right], \\
y & =\left[x_{1}, x_{3}\right]^{T}, \\
\lambda & =\frac{m}{\left(m+\left(I_{b} / r_{b}^{2}\right)\right)} .
\end{aligned}
$$

In the modeling process, some friction factors and some uncertainties are ignored. The uncertainties and the disturbances $D_{x}(t)$ and $D_{y}(t)$ are introduced into the subsystem. Then, the two linearized subsystems along the $x$-axis and $y$-axis are, respectively, expressed as follows:

$$
\begin{aligned}
& x:\left\{\begin{array}{l}
\dot{x}_{1}=x_{2}, \\
\dot{x}_{2}=-\lambda g u_{x}+D_{x}(t),
\end{array}\right. \\
& y:\left\{\begin{array}{l}
\dot{x}_{3}=x_{4}, \\
\dot{x}_{4}=-\lambda g u_{y}+D_{y}(t) .
\end{array}\right.
\end{aligned}
$$

The $X$-axis and $Y$-axis subsystems of the ball and plate system have the same mathematical model. Here, the controller is designed for the $X$-axis subsystem only. In the following, the proposed complementary nonsingular terminal sliding mode controller with a variable exponential power reaching law is used to realize the trajectory tracking experiment of the ball and plate system.

\subsection{Complementary Terminal Sliding Mode Control Based on} Variable Exponential Power Reaching Law. Compared with traditional sliding mode control, complementary sliding mode control can effectively reduce system tracking error and improve system tracking performance compared with traditional sliding mode control. However, compared with terminal sliding mode control, when the error is reduced, the convergence speed becomes slower. Therefore, combining complementary sliding mode control and terminal sliding mode control, a complementary terminal sliding mode control is proposed to improve the control performance of the system.

Define the tracking error of the system as $e=x_{x d}-x_{1}$, where $x_{x d}$ is the predetermined tracking trajectory of the ball and $x_{1}$ is the actual trajectory of the ball.

The traditional linear sliding surface is $s=c e+\dot{e}$, with $c>0$, a positive constant. Combining the complementary ideas and the singular problems of traditional terminal sliding mode, this paper proposes a complementary terminal sliding mode controller (CTSMC). The CTSMC adopts the design method of the integral sliding surface $S_{1}$ and complementary sliding surface $S_{2}$.

The integral sliding surface $S_{1}$ is defined as

$$
S_{1}=\dot{e}+2 c e+c e^{m}+c^{2} \int\left(e^{m}+e\right) \mathrm{d} t,
$$

where $c$ is a normal number, $0<m=(p / q)<1, p<q$, and both $p$ and $q$ are positive odd numbers.

The derivative of equation (24) along with equation (22) can be obtained as

$$
\begin{aligned}
\dot{S}_{1} & =\ddot{e}+2 c \dot{e}+c m e^{m-1} \dot{e}+c^{2}\left(e^{m}+e\right) \\
& =\ddot{x}_{\mathrm{xd}}-g_{x} u_{x}-D_{x}+2 c \dot{e}+c m e^{m-1} \dot{e}+c^{2}\left(e^{m}+e\right),
\end{aligned}
$$

where $g_{x}=-\lambda g$ and $D_{x}$ is the abbreviation of $D_{x}(t)$.

The complementary sliding surface $S_{2}$ is designed as

$$
S_{2}=\dot{e}+c e^{m}-c^{2} \int\left(e^{m}+e\right) \mathrm{d} t .
$$

The sum of two sliding surfaces is defined as $\sigma$, and the following relation can be obtained:

$$
\dot{S}_{2}+c \sigma=\dot{S}_{1}
$$

According to the system state equation, the CTSMC control law $u_{x}$ is designed. $u_{x}$ is composed of equivalent control law $u_{\text {xeq }}$ and switching control law $u_{\text {xh }}$, which is expressed as

$$
\begin{aligned}
u_{x} & =u_{x e q}+u_{x h}, \\
u_{x e q} & =\frac{1}{g_{x}}\left(\ddot{x}_{x d}+c\left(2 \dot{e}+m e^{m-1} \dot{e}+c\left(e^{m}+e\right)+S_{1}\right)-D_{x}\right),
\end{aligned}
$$

$$
u_{x h}=\frac{1}{g_{x}}\left(k_{1} \mathrm{fal}(\sigma, \alpha, \delta)+k_{2}|\sigma|^{\beta} \sigma\right) .
$$

In order to reduce the chattering of the sign function, the tanh function is used instead of the sign function. Equation (30) is updated as follows: 


$$
\begin{gathered}
u_{x h}=\frac{1}{g_{x}}\left(k_{1} \mathrm{fal}^{\prime}(\sigma, a, \delta)+k_{2}|\sigma|^{\beta} \sigma\right), \\
\operatorname{fal}^{\prime}(\sigma, a, \delta)= \begin{cases}|\sigma|^{a} \tanh (\sigma), \quad|\sigma|>\delta, \\
\frac{\sigma}{\delta^{1-a}}, & |\sigma| \leq \delta .\end{cases}
\end{gathered}
$$

According to the Lyapunov stability criterion, the Lyapunov function is selected as

$$
V=\frac{1}{2}\left(S_{1}^{2}+S_{2}^{2}\right)
$$

Therefore, from the derivation of equation (32), substitute equation (31) into

$$
\begin{aligned}
\dot{V} & =S_{1} \dot{S}_{1}+S_{2} \dot{S}_{2} \\
& =\left(S_{1}+S_{2}\right)\left(\ddot{x}_{x d}-g_{x} u_{x}-D_{x}+2 c \dot{e}+c m e^{m-1} \dot{e}+c^{2}\left(e^{m}+e\right)-c S_{2}\right) \\
& =-c\left(S_{1}+S_{2}\right)^{2}+\left(S_{1}+S_{2}\right)\left(-g_{x} u_{x h}\right) \leq 0 .
\end{aligned}
$$

In the formula, when $S_{1}+S_{2}>0, u_{x h}>0$; when $S_{1}+S_{2}<0, u_{x h}<0$. Therefore, the system is stable in the sense of Lyapunov.

4.3. Simulation Results. The mass of the ball $m=0.264 \mathrm{~kg}$, the radius $r=0.02 \mathrm{~m}$, the moment of inertia $I_{b}=$ $4.2 \times 10^{-5} \mathrm{~kg} \cdot \mathrm{m}^{2}$, and the acceleration of gravity $g=9.8 \mathrm{~m} / \mathrm{s}^{2}$, External interference $D_{x}(t)=0.1$ sint +0.1 cost . In order to ensure the fairness of comparisons in the simulation, some parameters adopt similar settings, $k=60, k_{1}=$ $40, k_{2}=60, \alpha=1.5, a=0.5, \delta=1, \Delta=0.4, \mu=10, w=$ $3, c=50, p=9$, and $q=11$. The control target of the simulation tracking trajectory is selected as a circular curve, and the expression of the curve is

$$
\left\{\begin{array}{l}
x_{\mathrm{xd}}=\cos \pi t \\
x_{\mathrm{yd}}=\sin \pi t
\end{array}\right.
$$

According to equation (34), $x_{x d}$ and $x_{y d}$ have a secondorder derivative. The initial position coordinates of the ball are $(0.5,0.5)$, the initial coordinates of the circular curve are $(1,0)$, and the simulation time is $2 \mathrm{~s}$.

4.4. Experiment 1: Comparative Experiment of Reaching Law. Using the traditional linear sliding mode surface, the variable exponential power reaching law method proposed in this paper is compared with the double-power combination function approach law approach [24], the traditional power reaching law approach [15], and the new exponential reaching law approach [16]. According to the different reaching laws, the sliding mode controller of the $x$-axis subsystem of the ball and plate system is designed, and the comparative simulations are completed. The simulation results are shown in Figures 3-8.

Fast variable exponential power reaching law sliding mode controller (SMC + VEPRL):

$$
u_{x 1}=g_{x}^{-1}\left(c \dot{e}+\ddot{x}_{\mathrm{xd}}-D_{x}(t)+k_{1} \mathrm{fal}^{\prime}(\sigma, \alpha, \delta)+k_{2}|\sigma|^{\beta} \sigma\right) .
$$

Double-power combination function reaching law sliding mode controller (SMC + DPRL):

$$
u_{x 2}=g_{x}^{-1}\left(c \dot{e}+\ddot{x}_{x d}-D_{x}(t)+k_{1} f a l(s, a, \delta)+k_{2}|s|^{b} \operatorname{sign}(s)\right) \text {. }
$$

Traditional power reaching law sliding mode controller $(\mathrm{SMC}+\mathrm{PRL})$ :

$$
u_{x 3}=g_{x}^{-1}\left(c \dot{e}+\ddot{x}_{\mathrm{xd}}-D_{x}(t)+k|s|^{b} \operatorname{sign}(s)\right) .
$$

Novel exponential reaching law sliding mode controller $(\mathrm{SMC}+\mathrm{ERL})$ :

$$
u_{x 4}=g_{x}^{-1}\left(c \dot{e}+\ddot{x}_{x d}-D_{x}(t)+\frac{k_{1}}{\left(\Delta+(1-\Delta) e^{-\mu|s|^{w}}\right)} \operatorname{sign}(s)\right) \text {. }
$$

In the reaching law comparison experiment, in the presence of interference, from the comparison of $x$-axis and $y$-axis tracking errors of the ball and plate system in Figures 3 and 4, the VEPRL method proposed in this paper has less error. The tracking error of the ERL method does not converge to zero, and the other methods have also good control performance. In Figures 5 and 6, the VEPRL method completes the trajectory tracking faster than the DPRL method at about $0.1 \mathrm{~s}$, while the other methods complete the tracking experiment after $0.15 \mathrm{~s}$. Therefore, the VEPRL method can complete the trajectory tracking faster under similar conditions. From Figures 7 and 8, the proposed reaching law can effectively weaken the chattering problem, while the SMC + ERL control law still has a relatively large chattering problem.

4.5. Experiment 2: Comparison Experiment of Sliding Mode Control Method. In this section, the reaching laws adopt the VEPRL method proposed in this paper. As shown in equation (1). Compare the complementary terminal sliding mode control method proposed in this paper with the traditional terminal sliding mode control (TSMC) method, complementary sliding mode control (CSMC) method, and traditional sliding mode control (SMC) method. According to the different sliding mode control methods, the sliding mode controller of the $x$-axis subsystem of the ball and plate system is designed, and the simulation comparison experiment is completed. The simulation results are shown in Figures 9-12.

Traditional terminal sliding mode controller $(\mathrm{TSMC}+\mathrm{VEPRL})$ :

$$
\begin{aligned}
s & =\dot{e}+c e^{m}, \\
u_{x 5} & =g_{x}^{-1}\left(c m e^{m-1} \dot{e}+\ddot{x}_{x d}-D_{x}(t)+k_{1} \mathrm{fal}^{\prime}(\sigma, a, \delta)+k_{2}|\sigma|^{\beta} \sigma\right) .
\end{aligned}
$$

Complementary sliding mode control (CSMC + VEPRL): 


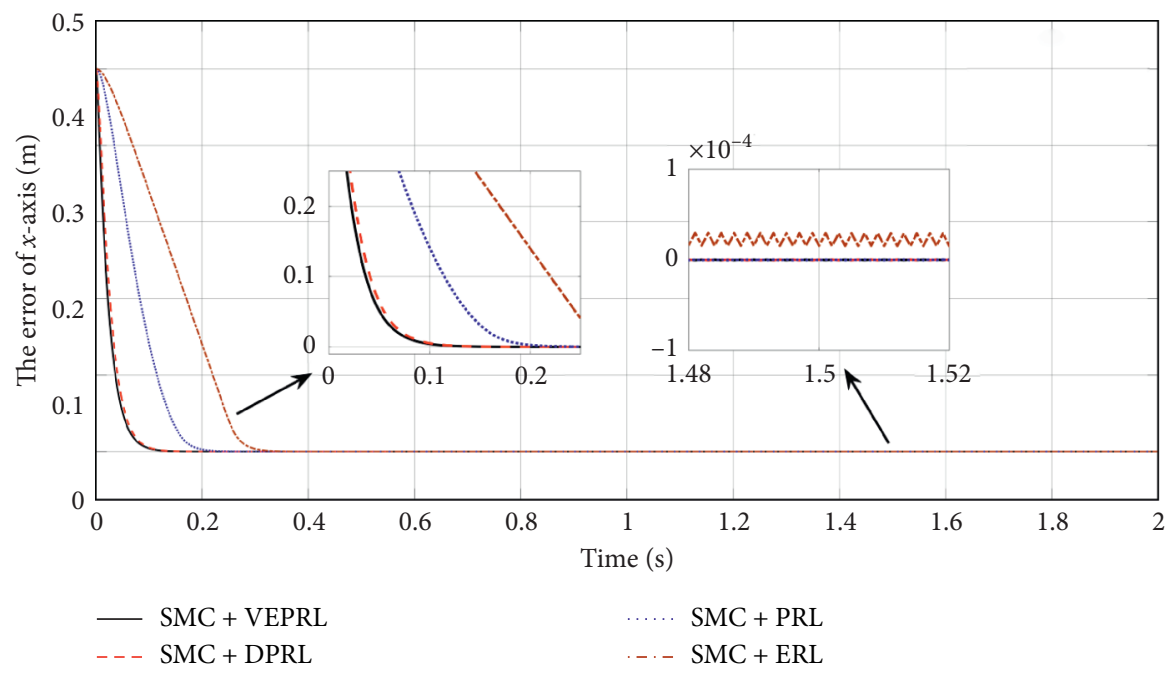

Figure 3: Comparison of $x$-axis tracking error of theball and plate system under SMC.



Figure 4: Comparison of $y$-axis tracking error of the ball and plate system under SMC.

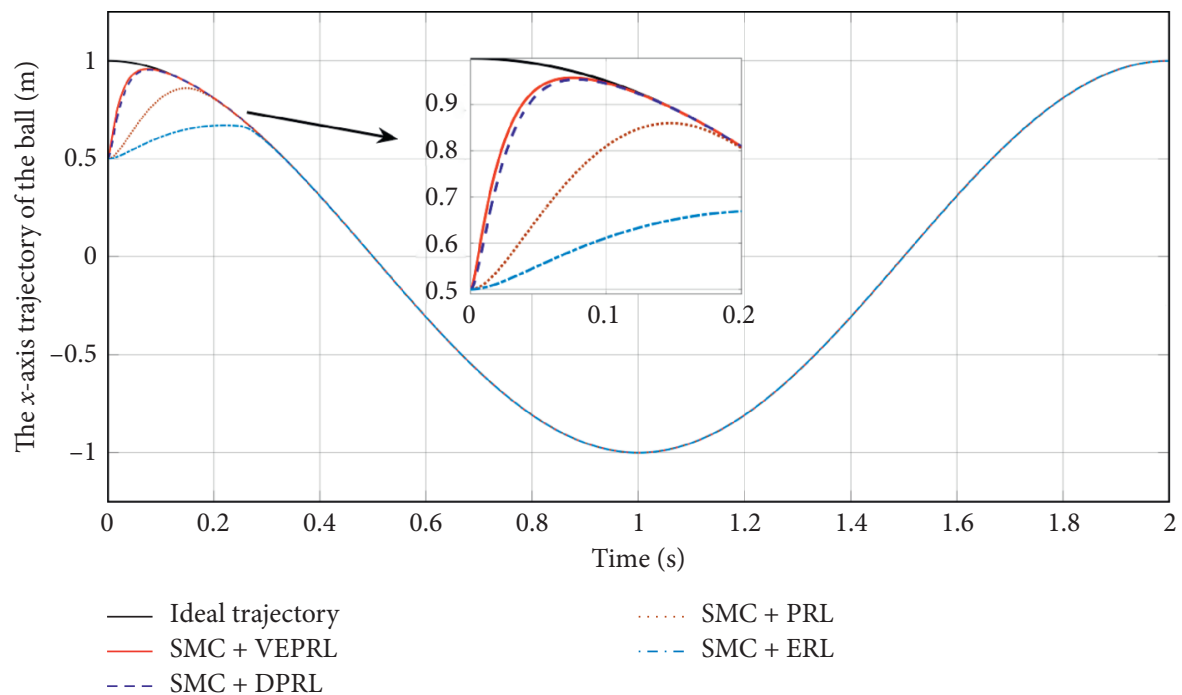

FIGURE 5: Comparison of $x$-axis tracking trajectory of the ball and plate system under SMC. 


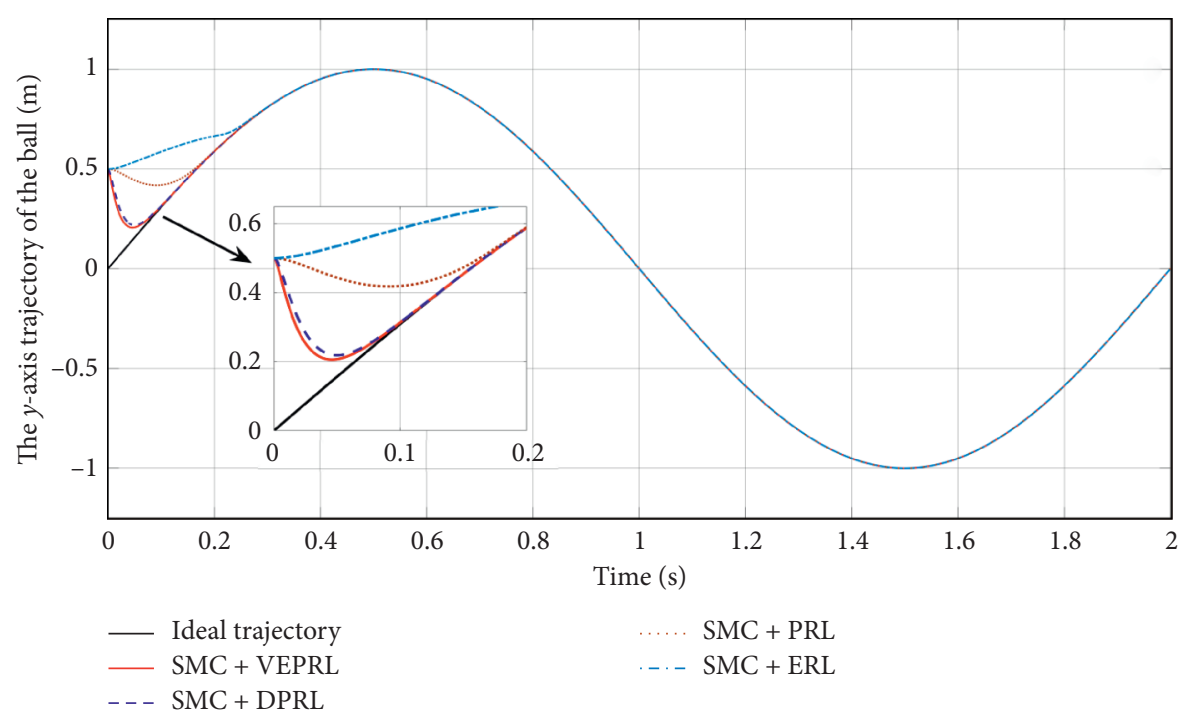

FIgURE 6: Comparison of $y$-axis tracking trajectory of the ball and plate system under SMC.

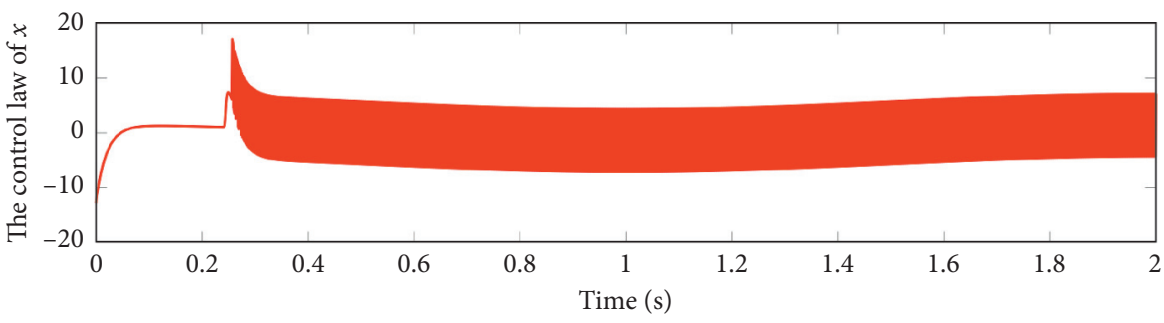

$-\mathrm{SMC}+\mathrm{ERL}$



- SMC + VEPRL
-- SMC + DPRL

Figure 7: The control law of $(x)$ under SMC.

$$
\begin{aligned}
S_{1}= & \dot{e}+2 c e+c^{2} \int e \mathrm{~d} t, \\
S_{2}= & \dot{e}-c^{2} \int e \mathrm{~d} t, \\
\sigma_{1}= & S_{1}+S_{2}, \\
u_{x 6}= & \frac{1}{g_{x}}\left(\ddot{x}_{x d}+c\left(2 \dot{e}+c e+S_{1}\right)\right. \\
& \left.-D_{x}+k_{1} \mathrm{fal}^{\prime}(\sigma, a, \delta)+k_{2} k_{2}|\sigma|^{\beta} \sigma\right) .
\end{aligned}
$$

The traditional sliding mode control (SMC + VEPRL) is shown in equation (35).

In the comparison with the sliding mode control method, from the $x$-axis and $y$-axis tracking error curves of the ball and plate system in Figures 9 and 10, the proposed CTSMC method has higher control accuracy and can maintain excellent control performance under different initial variables. When the error is large, the CSMC has a faster convergence speed, but when the error is small, the TSMC has a better convergence performance. Moreover, CTSMC + VEPRL control method has smoother steady-state error than other control methods. In Figures 11 and 12, the CTSMC has completed the desired trajectory tracking before $0.05 \mathrm{~s}$, so that 

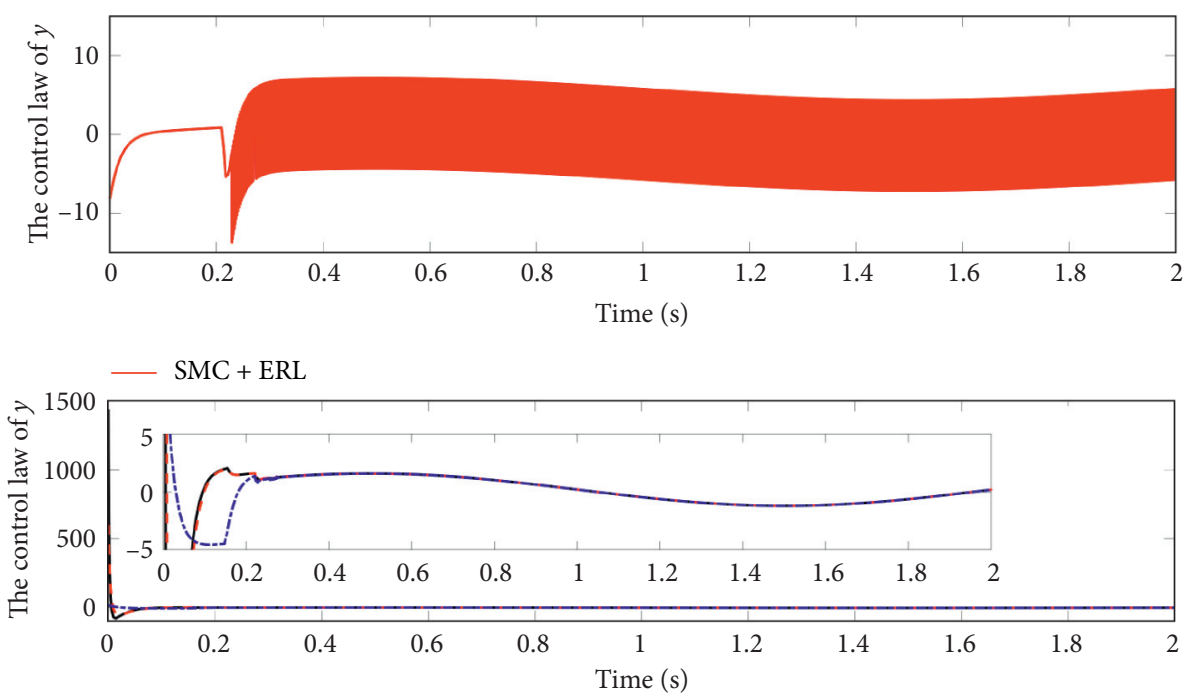

$-\mathrm{SMC}+\mathrm{VEPRL}$

- - SMC + DPRL

... SMC + PRL

FIgURE 8: The control law of $(y)$ under SMC.



FIGURE 9: Comparison of $x$-axis tracking error of the ball and plate system under VEPRL.

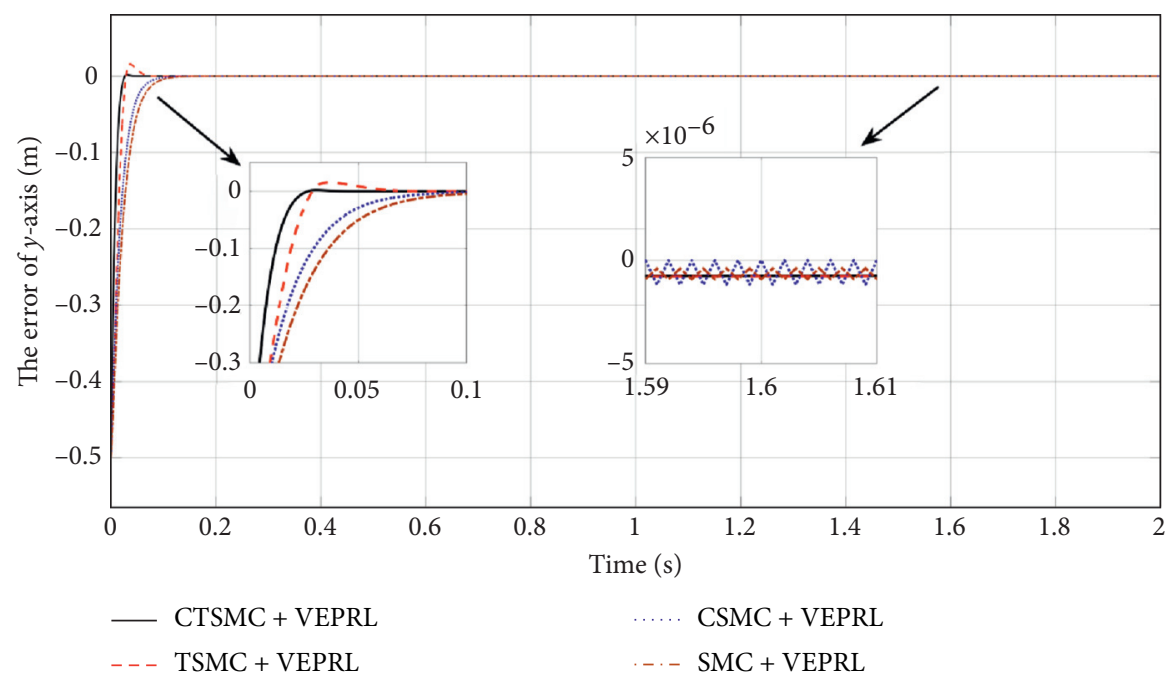

FIGURE 10: Comparison of $y$-axis tracking error of the ball and plate system under VEPRL. 




FIgURE 11: Comparison of $x$-axis tracking trajectory of the ball and plate system under VEPRL.

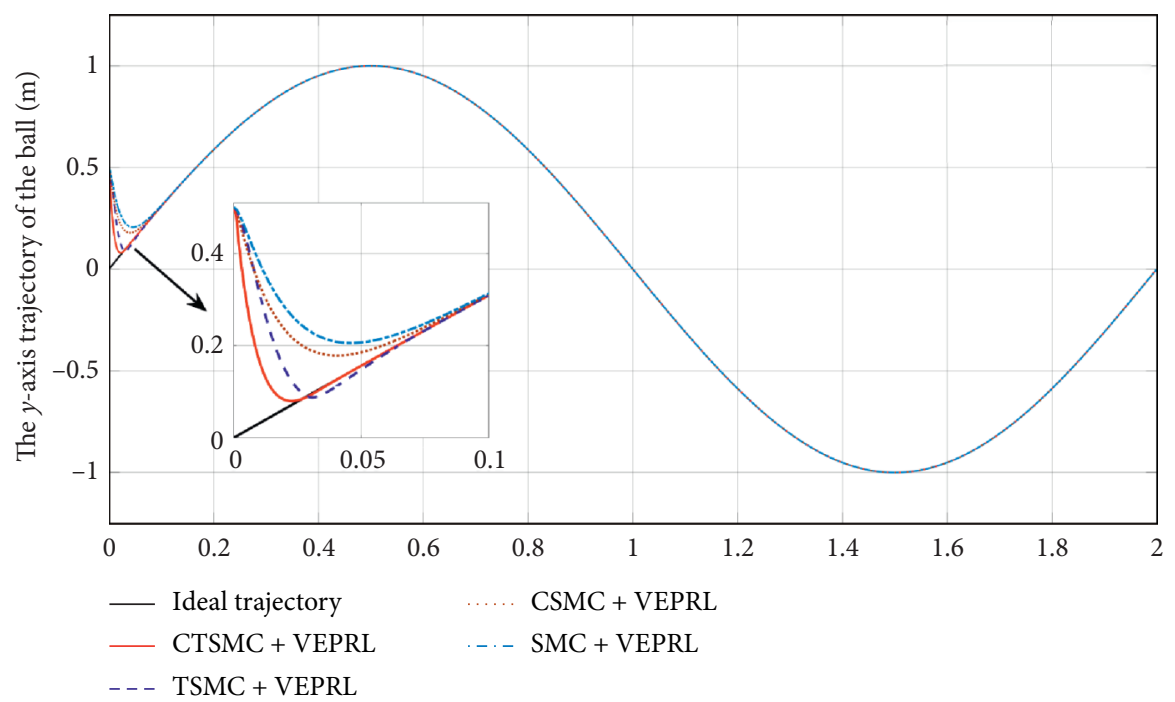

FIGURE 12: Comparison of $y$-axis tracking trajectory of the ball and plate system under VEPRL.

the CTSMC method has a faster system dynamic response performance. Therefore, the CTSMC + VEPRL control scheme proposed in this paper has better comprehensive control performance for tracking the circular curve in the ball and plate system control.

\section{Conclusion}

In this paper, a new variable exponential power reaching law and a complementary terminal sliding mode control method are proposed simultaneously. The characteristic analysis and theoretical proof of the reaching law are given. In the different approaching stages, the proposed reaching law has strong adaptive ability through combining a nonlinear combination function term and a variable power term. It can accelerate the approaching rate of system states far away from the sliding surface and effectively weaken the chattering phenomenon encountered in the sliding mode control. Combining the complementary idea, a complementary terminal sliding mode control is designed. It can make the system reach the equilibrium point with a faster convergence rate and has a better tracking performance. A new sliding mode control method is formed by combining the variable exponential power reaching law with the complementary terminal sliding mode control, which can enhance the dynamic response of the system. Moreover, the new sliding mode control scheme improves the tracking rate and control accuracy of the system. Simulation results verify the rationality and superiority of the proposed method. The proposed control method can be also applied to other nonlinear systems. Further works will focus on verifying the proposed sliding mode control scheme in practical applications. 


\section{Data Availability}

No data were used to support this study.

\section{Conflicts of Interest}

The authors declare that there are no conflicts of interest regarding the publication of this paper.

\section{Acknowledgments}

This work was supported in part by the National Natural Science Foundation of China under grant no. 51665025.

\section{References}

[1] W. Weibing Gao and J. C. Hung, "Variable structure control of nonlinear systems: a new approach," IEEE Transactions on Industrial Electronics, vol. 40, no. 1, pp. 45-55, 1993.

[2] S. Wang and J. Na, "Parameter estimation and adaptive control for servo mechanisms with friction compensation," IEEE Transactions on Industrial Informatics, vol. 16, no. 11, pp. 6816-6825, 2020.

[3] Y. Wang, Y. Xia, H. Li, and P. Zhou, "A new integral sliding mode design method for nonlinear stochastic systems," Automatica, vol. 90, pp. 304-309, 2018.

[4] F. Chen, R. Jiang, K. Zhang, B. Jiang, and G. Tao, "Robust backstepping sliding-mode control and observer-based fault estimation for a quadrotor UAV," IEEE Transactions on Industrial Electronics, vol. 63, no. 8, pp. 5044-5056, 2016.

[5] H. Li, P. Shi, and D. Yao, "Adaptive sliding-mode control of markov jump nonlinear systems with actuator faults," IEEE Transactions on Automatic Control, vol. 62, no. 4, pp. 19331939, 2017.

[6] R.-J. Lian, "Adaptive self-organizing fuzzy sliding-mode radial basis-function neural-network controller for robotic systems," IEEE Transactions on Industrial Electronics, vol. 61, no. 3, pp. 1493-1503, 2014.

[7] Q. P. Ha, D. C. Rye, and H. F. Durrant-Whyte, "Fuzzy moving sliding mode control with application to robotic manipulators," Automatica, vol. 35, no. 4, pp. 607-616, 1999.

[8] H.-Y. Chun-Hsien Tsai and F.-M. Yu, "Neuro-sliding mode control with its applications to seesaw systems," IEEE Transactions on Neural Networks, vol. 15, no. 1, pp. 124-134, 2004.

[9] O. Barambones and P. Alkorta, "Position control of the induction motor using an adaptive sliding-mode controller and observers," IEEE Transactions on Industrial Electronics, vol. 61, no. 12, pp. 6556-6565, 2014.

[10] S. Wang, J. Na, and Y. Xing, "Adaptive optimal parameter estimation and control of servo mechanisms: theory and experiments," IEEE Transactions on Industrial Electronics, p. $1,2020$.

[11] M. Defoort, T. Floquet, A. Kokosy, and W. Perruquetti, “A novel higher order sliding mode control scheme," Systems \& Control Letters, vol. 58, no. 2, pp. 102-108, 2009.

[12] J. Fei, N. Liu, and M. Hua, "Adaptive backstepping neural control of active power filter using complementary sliding mode approach," in Proceedings of the 2019 International Russian Automation Conference (RusAutoCon), pp. 1-6, Sochi, Russia, September 2019.

[13] Q. Chen, X. Yu, M. Sun, C. Wu, and Z. Fu, "Adaptive repetitive learning control of PMSM servo systems with bounded nonparametric uncertainties: theory and experiments," IEEE Transactions on Industrial Electronics, 2020.
[14] S. Wang, L. Tao, Q. Chen, J. Na, and X. Ren, "USDE-based sliding mode control for servo mechanisms with unknown system dynamics," IEEE/ASME Transactions on Mechatronics, vol. 25, no. 2, pp. 1056-1066, 2020.

[15] W. B. Gao, Theory and Design Method for Variable Sliding Mode Control, Science Press, Beijing, China, 1996.

[16] S. Yu, X. Yu, B. Shirinzadeh, and Z. Man, "Continuous finitetime control for robotic manipulators with terminal sliding mode," Automatica, vol. 41, no. 11, pp. 1957-1964, 2005.

[17] C. J. Fallaha, M. Saad, H. Y. Kanaan, and K. Al-Haddad, "Sliding-mode robot control with exponential reaching law," IEEE Transactions on Industrial Electronics, vol. 58, no. 2, pp. 600-610, 2011.

[18] H. Mei and Y. Wang, "Fast convergent sliding mode variable structure control of robot," Information and Control, vol. 35, no. 5, pp. 552-557, 2009.

[19] Y. Zhang, G. Ma, Y. Guo, and T. Zeng, "A multi power reaching law of sliding mode control design and analysis," Acta Automatica Sinica, vol. 42, no. 3, pp. 466-472, 2016.

[20] H. Ma, J. Wu, and Z. Xiong, "Discrete-time sliding-mode control with improved quasi-sliding-mode domain," IEEE Transactions on Industrial Electronics, vol. 63, no. 10, pp. 6292-6304, 2016.

[21] S. Juhng-Perng and C.-C. Wang, "Complementary sliding control of non-linear systems," International Journal of Control, vol. 75, no. 5, pp. 360-368, 2002.

[22] H. Jin and X. Zhao, "Complementary sliding mode control via elman neural Network for permanent magnet linear servo system," IEEE Access, vol. 7, pp. 82183-82193, 2019.

[23] V. Utkin, "Variable structure systems with sliding modes," IEEE Transactions on Automatic Control, vol. 22, no. 2, pp. 212-222, 1977.

[24] Y. Liao, Y. Yang, and Y. Wang, "Novel double power cfombination function reaching law for sliding mode control," Journal of National University of Defense Technology, vol. 42, no. 3, pp. 105-110, 2016.

[25] G. Michael Marks, Y. B. Shtessel, G. Harvey, and I. A. Shkolnikov, "Effects of high order sliding mode guidance and observers on hit-to-kill interceptions ," in Proceedings of the AIAA Guidance, Navigation, and Control Conference and Exhibit, San Francisco, CA, USA, August 2005. 